\section{Resistencia a la insulina, cortisol y composición corporal mediante dilución isotópica en niños costarricenses}

Valverde-Vindas $\mathrm{Al}^{1}$, Quintana-Guzmán $\mathrm{EM}^{2}$, Salas-Chaves $\mathrm{MP}^{3}$

Resumen

ANTECEDENTES: la obesidad infantil es un proceso que involucra en sí mismo un estrés oxidativo y un estado inflamatorio crónico que estimula la producción de corticosteroides, promueve la aparición temprana de resistencia insulínica y de enfermedades como la diabetes mellitus tipo 2.

OBJETIVO: evaluar la composición corporal en población infantil costarricense mediante técnicas de dilución isotópica con deuterio y analizar su relación con el índice de resistencia a la insulina y niveles de cortisol sérico matutino.

MATERIALES Y MÉTODOS: determinación de cortisol sérico matutino y resistencia a la insulina mediante el método homeostático en 113 niños y niñas escolares. Se realiza la comparación por grupos según la clasificación McCarthy para porcentaje de masa grasa, determinada mediante el método de dilución isotópica.

RESULTADOS: no se observan diferencias estadísticamente significativas entre los grupos de masa grasa, según la clasificación McCarthy para las variables de cortisol sérico matutino e índice HOMA (modelo homeostático) de resistencia a la insulina, en la muestra seleccionada.

CONCLUSIÓN: en niños y niñas entre 6 y 9 años de edad no se evidencian las características bioquímicas que se esperan del síndrome metabólico, dada la poca exposición temporal al estrés oxidativo que produce la obesidad, determinada por medio de la medición de masa grasa con un método temprano como la dilución isotópica. Asimismo, en edades tempranas los mecanismos de detoxificación renal de los corticosteroides son suficientes para evitar su elevación sérica, protegiendo al niño de sus efectos a largo plazo.

PALABRAS CLAVE: composición corporal, deuterio, HOMA, resistencia insulina, cortisol, espectrometría infrarroja.
${ }^{1}$ Sistema de Estudios de Posgrado. ²Departamento de Análisis Clínicos. ${ }^{3}$ Departamento de Microbiología. Facultad de Microbiología, Universidad de Costa Rica.

Recibido: 27 de octubre del 2015

Aceptado: 11 de febrero del 2016

Correspondencia

Dr. Allan Valverde Vindas

Sistema Estudios de Posgrado

Universidad de Costa Rica

allan.valverdevindas@ucr.ac.cr

Este artículo debe citarse como Valverde-Vindas AI, Quintana-Guzmán EM, SalasChaves MP. Resistencia a la insulina, cortisol y composición corporal mediante dilución isotópica en niños costarricenses. Acta Pediatr Mex 2016;37(3):139-148. 
Acta Pediatr Mex. 2016 May;37(3):139-148.

\section{Insulin resistance, cortisol and body composition by isotope dilution in costa rican children.}

Valverde-Vindas $\mathrm{Al}^{1}$, Quintana-Guzmán $\mathrm{EM}^{2}$, Salas-Chaves $\mathrm{MP}^{3}$

Abstract

INTRODUCTION: Childhood obesity is a condition which involves in itself an oxidative stress and chronic inflammation that stimulates the production of corticosteroids and promotes the early onset of insulin resistance and diseases such as type 2 diabetes mellitus.

OBJECTIVE: Assess ment of body composition in Costa Rican child population using deuterium dilution techniques and analysis its of relationship with the index of insulin resistance and morning serum cortisol levels.

RESULTS: No statistically significant differences between groups of fat mass classification according to McCarthy was observed for variables morning serum cortisol and HOMA index of insulin resistance in the selected sample.

CONCLUSION: In children between 6 and 9 years old the expected biochemical characteristics of the metabolic syndrome are not evident, given the short time exposure to oxidative stress of obesity, determined by measuring fat mass with an early method as isotopic dilution. Also, in early ages renal detoxification mechanisms of corticosteroids are sufficient to prevent elevated serum concentration and protect children from its long-term effects.

KEYWORDS: body composition; deuterium; HOMA; insulin resistance; cortisol; children; infrared spectrometry

\author{
${ }^{1}$ Sistema de Estudios de Posgrado. \\ 22Departamento de Análisis Clínicos. \\ ${ }^{3}$ Departamento de Microbiología. \\ Facultad de Microbiología, Universidad de \\ Costa Rica. \\ Correspondence \\ Dr. Allan Valverde Vindas \\ Sistema Estudios de Posgrado \\ Universidad de Costa Rica \\ allan.valverdevindas@ucr.ac.cr
}

\section{INTRODUCCIÓN}

América Latina se encuentra en una transición epidemiológica en la que cada vez son menos los casos de infección y desnutrición, dando paso a la obesidad, que correlacionada con las enfermedades crónicas, se está convirtiendo en un factor determinante de morbilidad y mortalidad.

La población pediátrica costarricense no es la excepción, dada la incidencia general de diabetes mellitus del año 2006, de 3.44 por cada 100,000 menores de 15 años de edad, siendo más frecuente en la Meseta Central con ligero predominio del sexo femenino (53\%) sobre el masculino $(47 \%)$, con edad promedio de presentación de 8 años y 6 meses. ${ }^{1}$ Asimismo, en una población de 115 niños diabéticos diagnosticados en el Hospital Nacional de Niños entre 2006 y 2008, 16.5\% fueron clasificados como diabéticos tipo 2 (DM2), demostrándose que el incremento de casos de DM2 en niños sigue manteniéndose. ${ }^{2}$ 
La obesidad conduce a un estado de inflamación crónica relacionada con el estrés oxidativo, que a su vez está condicionado con algunos micronutrientes. ${ }^{3}$ En condiciones normales el cortisol contrarresta la acción de la insulina contribuyendo a la hiperglicemia y estimulando la gluconeogénesis. ${ }^{4}$ Sin embargo, en condiciones de estrés oxidativo sostenido, los niveles de hormona liberadora de corticotropina $(\mathrm{CRH})$ se mantienen elevados, lo cual tiene dos efectos: por un lado reduce los niveles de somatomedina y gonadotropinas, con lo cual se genera resistencia a la insulina y pérdida de masa magra y por otro lado mantiene los niveles de cortisol persistentemente elevados. Esta elevación se asocia con un aumento en la secreción de insulina, lo que conduce a sinergia de la acción lipogénica de éstos sobre la grasa visceral; aunque con respecto a otras acciones sean antagonistas. ${ }^{5}$ Este desequilibrio metabólico general predispone al paciente a lo que actualmente se conoce como el síndrome metabólico, que se caracteriza por reunir padecimientos como hipertensión, ateroesclerosis, disminución de lipoproteínas de alta densidad, hipertrigliceridemia y, por supuesto, más obesidad. ${ }^{5}$

El peso corporal y la relación peso para la talla son indicadores clave para la evaluación nutricional, tanto para población como individual. Sin embargo, para el diagnóstico de sobrepeso y obesidad se requiere demostrar un incremento de la grasa corporal. El método isotópico de dilución con deuterio se considera como de referencia para estimar composición corporal. ${ }^{6}$ En este caso, se mide el agua corporal total basándose en principios de dilución en muestras de saliva, sangre u orina utilizando espectrometría de masas o espectrometría infrarroja. ${ }^{7}$

\section{OBJETIVO}

Evaluar la composición corporal en población infantil costarricense mediante técnicas de dilu- ción isotópica con deuterio y analizar su relación con el índice de resistencia a la insulina y los niveles de cortisol sérico matutino.

\section{MATERIALES Y MÉTODOS}

Población en estudio

El estudio incluyó 113 niños y niñas costarricenses en etapa prepúber, con edades entre 6 y 9 años. Se tomaron muestras de saliva y sangre para determinar su composición corporal, niveles de cortisol, insulina y glucosa séricos y el índice de resistencia a la insulina.

\section{Procedimientos éticos}

Un Formulario de Consentimiento Informado, escrito previamente y aprobado por el Comité Ético-Científico de la Vicerrectoría de Investigación de la Universidad de Costa Rica, fue distribuido a todos los individuos y aprobado y firmado por los padres de familia, como requisito indispensable para su participación.

\section{Variables de composición corporal}

En ayuno de dos horas se obtuvieron dos muestras de saliva, una basal a tiempo cero y una segunda a las tres horas posdosis (agua con deuterio), que fueron analizadas con el espectrofotómetro infrarrojo transformado de Fourier (FTIR) Shimadzu, modelo IRPrestige-21 NIR, para determinar la composición corporal de los individuos por medición de deuterio.

\section{Variables bioquímicas}

Para el análisis de glicemia, cortisol e insulina los niños se presentaron en una segunda sesión con ayuno de ocho horas y se obtuvieron por punción venosa $10 \mathrm{~mL}$ de sangre total en tubos sin anticoagulante para obtener suero. 
Los niveles de glucosa sérica se determinaron empleando el método colorimétrico enzimático usando glucosa oxidasa de la casa comercial Alfa-Wassermann, modelo ACE ${ }^{\circledR}$. Para la determinación de insulina en suero se empleó el método de placas de ELISA indirecto DRG Diagnostics ${ }^{\circledR}$.

La resistencia a insulina se determinó con el modelo homeostático (HOMA-IR), el cual se expresa como el índice de resistencia a la insulina (IRI) calculado multiplicando la concentración de insulina sérica $(\mu \mathrm{UI} / \mathrm{mL})$ por la concentración de glucosa en ayunas $(\mathrm{mmol} / \mathrm{L})$ dividiendo el resultado entre 22.5.

En la determinación de cortisol en suero se empleó el equipo Immulite ${ }^{\circledR} 2000$, el cual sigue la metodología de ELISA, automatizado tipo competitivo de la casa comercial SIEMENS ${ }^{\circledR}$.

\section{Análisis de los datos}

El análisis de los datos se realizó con el sistema informático SigmaPlot para Windows, versión 11.0, mediante el cual se evaluó la normalidad de los parámetros utilizando la prueba estadística de Shapiro-Wilk.

Los datos se presentan como la media \pm desviación estándar (DE) y los valores superiores a la media de \pm 3 DE fueron eliminados como valores atípicos.

Para detectar diferencias estadísticamente significativas entre los grupos se empleó la prueba estadística ANDEVA de una vía, para las variables de distribución simétrica y análisis Kruskal-Wallis de varianza de una vía en rangos para las variables de distribución asimétrica. Además, se utilizó la correlación de Spearman para determinar la existencia de correlación lineal. Un valor de $\mathrm{p}<0.05$ fue considerado para indicar normalidad y significancia estadística.

\section{RESULTADOS}

Se analizaron los resultados de 60 niños y 53 niñas con una edad promedio de $7.30 \pm 0.40$ años. La población en estudio se agrupó según la clasificación de McCarthy y sus colaboradores $(2006)^{8}$ para porcentaje de grasa corporal, de manera tal que $10.6 \%$ de los niños se hallaron en el grupo infragrasa, $27.7 \%$ normograsa, $12.4 \%$ sobregrasa y $51.3 \%$ obesos.

$\mathrm{Al}$ analizar el comportamiento de las diferentes variables en estudio por grupos de porcentaje de grasa no hubo diferencias estadísticamente significativas entre ninguno. Sin embargo, se observaron aumentos en la cortisolemia del grupo normograsa con respecto al grupo infragrasa; de igual manera, aunque en menor magnitud, el cortisol sérico en promedio fue mayor en el grupo de niños obesos con respecto al grupo de niños con sobregrasa. El grupo con la mayor cortisolemia promedio fue el normograsa (223.1 nmol/L), mientras que el grupo sobregrasa presentó la menor concentración promedio de cortisol sérico (196.75 nmol/L) (Figura 1).

La concentración de glucosa sérica en ayunas del grupo infragrasa alcanzó los mayores niveles promedio $(4.66 \mathrm{mmol} / \mathrm{L})$, mientras que el grupo sobregrasa mostró la menor concentración $(4.46 \mathrm{mmol} / \mathrm{L})$. Hubo un ligero descenso en la concentración sérica de la glucosa entre los grupos infragrasa, normograsa y sobregrasa, con una ligera elevación al final en el grupo de niños con obesidad.

El grupo infragrasa tuvo la mayor concentración de insulina sérica $(9.15 \mu \mathrm{UI} / \mathrm{mL})$, en comparación con el grupo normograsa que presentó la menor concentración promedio $(7.22 \mu \mathrm{Ul} / \mathrm{mL})$. Para esta variable se encontró una diminución entre el grupo infragrasa y el normograsa, seguido de una elevación en el grupo sobregrasa que se mantiene en magnitud semejante para el grupo con obesidad. 

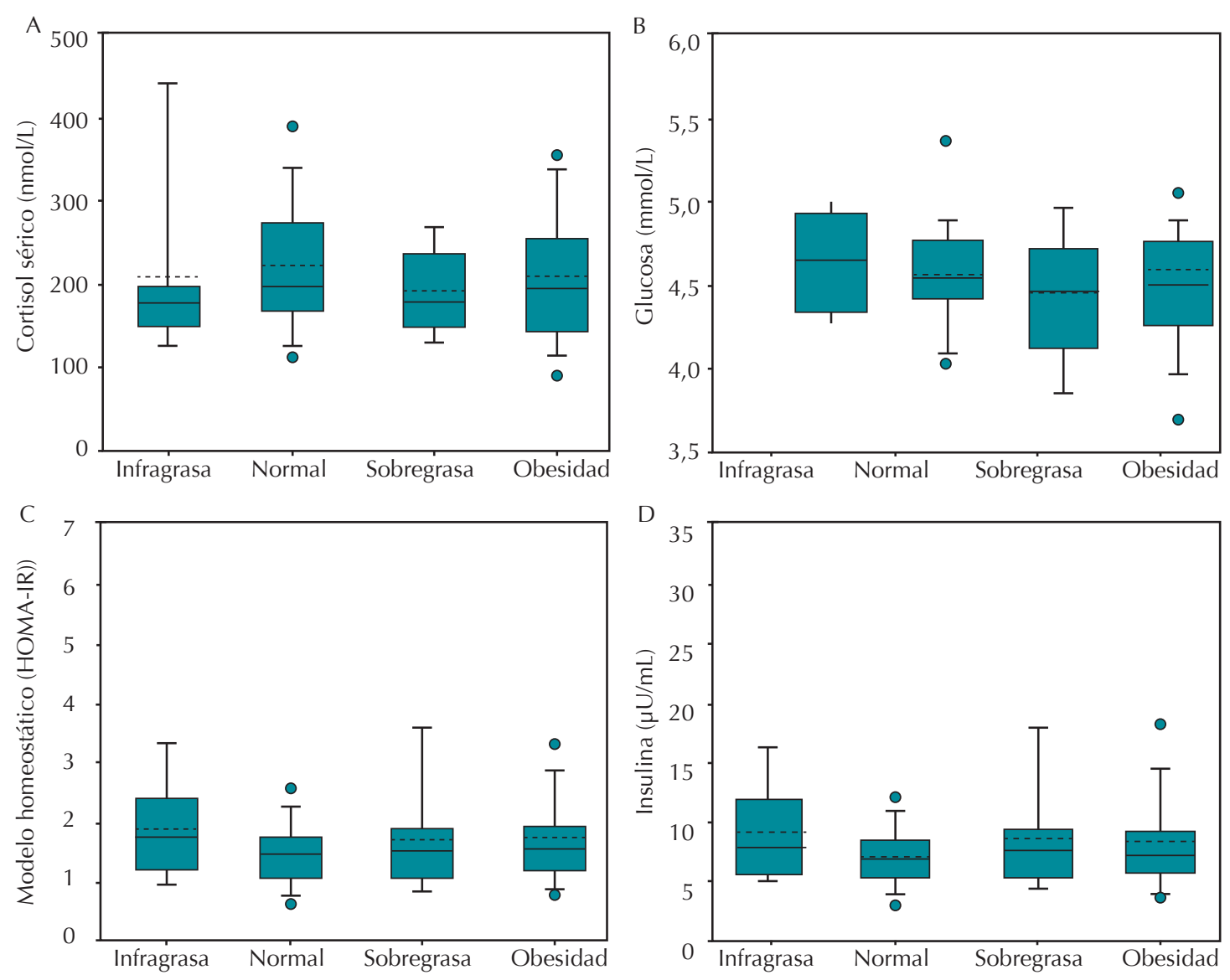

Figura 1. Gráfico de cajas de cada variable según los grupos de grasa corporal. A) Gráfico comparativo del cortisol sérico promedio entre los grupos según grasa corporal. B) Gráfico comparativo de la glicemia promedio entre los grupos según grasa corporal. C) Gráfico comparativo del índice HOMA promedio entre los grupos según grasa corporal. D) Gráfico comparativo de la insulina sérica promedio entre los grupos según grasa corporal.

El índice homeostático para la resistencia a la insulina (HOMA-IR) mostró un comportamiento similar al presentado por la insulina sérica, de modo que el grupo normograsa tuvo el menor índice promedio (1.48) y el grupo infragrasa el mayor índice promedio (1.89), mientras que los grupos sobregrasa y obesidad se mantuvieron semejantes.

Al analizar el coeficiente de correlación de Spearman $(\rho)$ para las diferentes variables con- tra el porcentaje de grasa corporal se vio que no existe una correlación lineal en ninguno de los parámetros en estudio, pues los valores del estadístico $\rho$ de Spearman no se aproximan lo suficiente a la unidad y $\mathrm{p}$ fue mayor al valor crítico con una significancia de 0.050 en todos los casos, aceptándose la hipótesis nula $\left(\mathrm{H}_{0}: \rho=\right.$ $0)$. Sin embargo, al considerar las rectas de mejor ajuste para cada una de las variables, se notó que había una ligera tendencia hacia la disminución en la concentración de cortisol conforme 
aumentaba el porcentaje de grasa corporal; de igual manera ocurre con la glicemia. Mientras que en el caso de la insulinemia y el índice homeostático para la resistencia a la insulina, no se observó ningún cambio con respecto al porcentaje de grasa corporal.

\section{DISCUSIÓN}

La determinación del porcentaje de masa grasa por el método de dilución isotópica es un marcador temprano de obesidad, pues algunos estudios señalan que existe considerable cantidad de falsos negativos al emplear el índice de masa corporal como único indicador. Esto se debe a que el éste solamente considera el peso y la talla del individuo y pasa por alto la condición de individuos con un porcentaje de masa grasa superior a lo deseable, pero que aún no se hace evidente en el peso corporal. ${ }^{9}$ Por lo tanto, es posible que al emplear la clasificación de McCarthy y su grupo ciertos sujetos incluidos en el grupo "obesos" se encuentren en estados incipientes de obesidad y estrés oxidativo, razón por la cual no se aprecian diferencias significativas entre las variables bioquímicas de los grupos ni un patrón contundente de alteraciones hormonales debidas a dicha obesidad.

El estrés oxidativo se ha encontrado en niños obesos a nivel celular. Se ha sugerido que dicha obesidad podría ser un trastorno que promueve el estrés oxidativo crónico originado por un desequilibrio entre radicales tisulares libres, especies reactivas de oxígeno (ROS) y antioxidantes. ${ }^{10}$ Los antioxidantes sanguíneos como carotenoides, vitaminas $\mathrm{E}$ y $\mathrm{C}$ y rastros de minerales se han encontrado consistentemente más bajos en niños y adultos obesos al compararlos con análogos no obesos. ${ }^{3}$ Sin embargo, para evidenciar las características bioquímicas séricas típicas del síndrome metabólico es necesaria una exposición crónica a dicho desequilibrio oxidativo. La corta edad de los individuos participantes en este estudio y los niveles de insulina, cortisol y glucosa séricos obtenidos permiten intuir que al no haber permanecido suficiente tiempo sometidos en dicho estado prooxidante, los efectos bioquímicos del mismo aún no son evidentes, siendo estos niveles independientes del porcentaje de masa grasa.

La acción general del cortisol sobre la glicemia se refleja en los resultados obtenidos, pues así como pacientes con síndrome de Cushing frecuentemente presentan niveles elevados de glucosa, en pacientes con el síndrome metabólico los niveles de cortisol están significativamente asociados con la concentración de glucosa en ayunas. La relación entre la hiperglicemia en ayunas y el cortisol se debe al efecto de los glucocorticoides en la gluconeogénesis hepática y la secreción de insulina. ${ }^{11}$ Es posible observar cómo los grupos con mayor cortisolemia (infragrasa y normograsa) también tuvieron mayor glicemia, al contrario de los grupos con menor cortisolemia (sobregrasa y obesidad).

El síndrome de Cushing se caracteriza por una redistribución del tejido adiposo de sitios periféricos a sitios centrales del cuerpo, principalmente en la región del tronco y los depósitos viscerales. De manera similar, la obesidad central es uno de los componentes más importantes del síndrome metabólico. El cortisol parece jugar un rol en la adiposidad en este síndrome, promoviendo la proliferación y diferenciación de los adipocitos humanos, ya que sus receptores son más abundantes en el tejido visceral. ${ }^{12}$

Se ha descrito un incremento en la excreción urinaria de cortisol libre en pacientes con el síndrome metabólico, aunque los estudios realizados no logran confirmar este hallazgo al comparar la excreción urinaria de cortisol con la grasa total en mujeres de edad media. Sí se observó un incremento en la cortisona/cortisol urinario en las mujeres con mayor grasa abdominal en comparación con las que tenían una distribución periférica de la distribución grasa, lo cual sugiere un incremento en el metabolismo del cortisol. ${ }^{13}$ 
En general parece que una hipercortisolemia relativa podría contribuir al desarrollo de las diferentes características del síndrome metabólico. Sin embargo, no está claro si los glucocorticoides juegan un papel en la patogénesis de la obesidad, puesto que los niveles circulantes de cortisol no siempre se encuentran elevados en sujetos obesos, tal como se evidencia en el presente estudio. En algunos estudios aparecen niveles de cortisol sérico menores en sujetos obesos que en los delgados, ${ }^{14-16}$ aspecto en el que coincidimos con los resultados de esta investigación.

Este hecho podría ser parcialmente explicado por el aclaramiento metabólico incrementado del cortisol debido a que los pacientes obesos muestran incrementada la excreción de cortisol libre y sus metabolitos. ${ }^{13,16}$ Existen otros determinantes además de los niveles circulantes de cortisol, como la expresión local de la enzima $11 \beta$-hidroxiesteroide deshidrogenasa (11 $\beta$-HSD), la cual parece jugar un papel importante en la interacción entre cortisol y adiposidad. La $11 \beta$-HSD cataliza la conversión de la hormona activa C11-hidroxiglucocorticoide (cortisol, corticosterona) a su forma inactiva C11-ceto metabolitos: cortisona, 11-dehidrocosticosterona. ${ }^{17,18}$

Hay dos isoformas de la $11 \beta$-HSD: $11 \beta$-HSD2 es una deshidrogenasa dependiente de difosfato de adenosina (ADP) de alta afinidad que protege el receptor de mineralocorticoides en los túbulos renales de la estimulación excesiva del cortisol. Esta isoforma ha sido identificada también en colon, glándulas salivales y placenta. ${ }^{17,18}$ Mutaciones en el gen que codifica por la $11 \beta$-HSD2 causan una forma de hipertensión arterial heredada, el síndrome de "exceso aparente de mineralocorticoides", en el cual el cortisol actúa como un potente mineralocorticoides. ${ }^{19}$ Más aún, la deleción homocigota de la $11 \beta$-HSD2 en ratones causa hipertensión e hipokalemia, aunado a niveles suprimidos de renina y aldosterona séricos. ${ }^{18} \mathrm{La}$ hipertensión que puede observarse en 85-90\% de pacientes con síndrome de Cushing, indica una posible saturación de la enzima por el exceso de cortisol circulante. ${ }^{20}$ Sin embargo, en el caso de niños obesos en el presente estudio no tenían niveles de hipercortisolemia (a diferencia de los pacientes con síndrome de Cushing), tomando en consideración que se trata de niños sanos y que el catabolismo renal de dicha hormona se lleva a cabo de una forma normal. Se esperaría que los niveles más bajos de cortisol sérico de los grupos sobregrasa y obesidad, comparados con los grupos infragrasa y normograsa, estén dados por la acción incrementada de la enzima $11 \beta-H S D 2$, como parte de su función renal protectora.

Otros estudios mencionan que el aclaramiento del cortisol aparece inversamente correlacionado con la sensibilidad a la insulina y esta correlación es independiente de la grasa corporal. ${ }^{21}$ Dichos hallazgos respaldan los resultados encontrados en el presente estudio, pues al comparar los valores de cortisol e índice homeostático para la resistencia a la insulina por grupos de grasa corporal, se observa que el grupo normograsa fue el que tuvo la mayor cortisolemia y al mismo tiempo el menor índice homeostático para la resistencia a la insulina. Esto permite inferir que al haber un menor aclaramiento de cortisol hay una mayor sensibilidad a la insulina, o dicho de otra manera, una menor resistencia (HOMA bajo). Lo interesante de este hallazgo es que al comparar los demás grupos se aprecia de igual manera cómo al disminuir el cortisol sérico aumenta la resistencia a la insulina, comportamiento que ocurre tanto en los grupos infragrasa, sobregrasa como obesidad; de manera que respalda el hecho de que dicho comportamiento es independiente del porcentaje de grasa corporal.

Los niveles de cortisol (urinario libre y sérico durante la noche), también se han asociado con resistencia a la insulina (analizado con el índice homeostático). ${ }^{22-24}$ Concentraciones altas 
de cortisol fueron relacionadas a una secreción reducida de insulina, un hallazgo consistente con datos in vitro e in vivo que muestran que los glucocorticoides regulan la secreción de insulina.

Unos investigadores emplearon ratones transgénicos con sensibilidad incrementada a los glucocorticoides restringida a las células beta del páncreas, mediante la sobreexpresión del receptor para glucocorticoides (GR), bajo control del promotor de la insulina. Al aplicar una prueba de tolerancia a la glucosa intravenosa a dichos animales de experimentación y compararlos con animales control, se encontró que los ratones modificados genéticamente tuvieron niveles de glicemia normales en ayunas y poscarga, mostrando menor tolerancia a la glucosa en comparación con los animales control. Al medir la insulina, 5 minutos después de aplicar la glucosa intravenosa, se observó un descenso en la respuesta aguda a la insulina en los ratones transgénicos. Estos resultados demostraron que los glucocorticoides inhiben directamente la liberación de la insulina in vivo e identifican a las células beta del páncreas como blancos importantes para la acción diabetogénica de los glucocorticoides. ${ }^{25}$ Así lo reflejan los resultados obtenidos en donde el grupo con mayor nivel de cortisol fue el que tuvo simultáneamente el menor nivel de insulina sérica e inversamente, los grupos con menor cortisolemia tuvieron mayor secreción de insulina.

Además, estudios realizados en niños obesos con o sin resistencia a la insulina según el modelo homeostático, muestran que la reducción del peso corporal reduce tanto los niveles de cortisol como la resistencia a la insulina en el grupo con resistencia a la insulina. Esto no se vio en niños sin resistencia a la insulina. ${ }^{23}$

\section{CONCLUSIÓN}

No se evidencia correlación entre el porcentaje de masa grasa, los niveles de cortisol sérico matutino y el índice homeostático para la resis- tencia a la insulina en la muestra seleccionada de niños entre 6 y 9 años de edad; sin embargo, cada vez son más los casos de niños obesos y diabéticos en nuestro medio. Intervenciones tempranas en el estilo de vida de estos niños y niñas enfocándose en el tratamiento de la grasa corporal y el incremento de la actividad física, son dos frentes que deberían ser prioritarios en el aspecto de salud pública, los cuales deberían ser promovidos en los diferentes entornos en que se desenvuelven los niños para prevenir de esta manera el desarrollo futuro de diabetes mellitus tipo 2 y otras patologías asociadas.

El tamizaje temprano de resistencia a la insulina empleando índices tales como el índice homeostático para la resistencia a la insulina en escolares, debería incluirse en el nivel primario de atención en salud como una herramienta para identificar tempranamente alteraciones en la glicemia y metabolismo de los carbohidratos y de esta manera evitar el progreso y las consecuencias de la enfermedad.

Se recomienda, para estudios experimentales posteriores, incluir biomarcadores de estrés oxidativo como vitamina A, C, E, actividad catalasa o hidroperóxidos plasmáticos ${ }^{26}$ para evaluar el estado antioxidante de los individuos, de manera que se pueda determinar si los niños obesos se encuentran en un estado oxidativo comparable con no obesos. Además, se recomienda obtener muestras de orina para la medición de cortisol y sus metabolitos, con el objetivo de determinar el aclaramiento de cortisol en los niños de manera que permita deducir si existe relación entre el aclaramiento de la hormona y el porcentaje de masa grasa de los niños. También sería deseable considerar la determinación de otras hormonas contrarreguladoras en el metabolismo de los carbohidratos de importancia para la edad. Por ejemplo, la hormona del crecimiento como colaboradora en el estado de la glicemia, dada su acción 
Valverde-Vindas Al et al. Resistencia a la insulina, cortisol y composición corporal en niños

hiperglicemiante durante el crecimiento y desarrollo en la infancia. ${ }^{27}$

\section{Agradecimientos}

A la Organización Internacional de Energía Atómica, por su auspicio y patrocinio, especialmente en la figura del profesor Eleuterio Umpiérrez, quién fue tutor experimental.

A la Vicerrectoría de Investigación de la Universidad de Costa Rica por el aporte económico para realizar este estudio.

A la Caja Costarricense del Seguro Social, representada en el Laboratorio de Medicina Nuclear del Hospital San Juan de Dios, que patrocinó este trabajo de investigación.

\section{REFERENCIAS}

1. Mora E. Diabetes infantil en Costa Rica [Internet]. San José (CR): 2011 [citado 22 de enero del 2013]. Disponible en: http://www.diabetesbajocontrol.com/.

2. Morales K, Richmond E, Bogarín R, Jaramillo O. Análisis de las hemoglobinas glucosiladas de los pacientes diabéticos diagnosticados del 2006 al 2008 en el Hospital Nacional de Niños. Acta Med Costarric. 2011;53(2):88-92.

3. Sallas T. Childhood Obesity: Effects of Micronutrients, Supplements, Genetics and Oxidative Stress. J Nurse Pract. 2011;7(8):647-653.

4. Boron W, Boulpaep E. Medical Phisiology A Cellular and Molecular Approach. 2arev. ed. Philadelphia: SAUNDERS ELSEVIER; 2009. 1337p.

5. Méndez J, Flores J, Noyola D, Mendoza E, Calderón J, Aradillas C. Asociación del índice de resistencia a la insulina con niveles de cortisol y medidas antropométricas por género de niños mexicanos en edad escolar. Bioquimia. 2007;32(4):126-133.

6. Salazar G, Rocha M, Mardones, F. ¿Es útil la antropometría para estimar la composición corporal en niños preescolares? Rev Chil Pediatr. 2003;74(1):37-45.

7. Ramírez $\mathrm{E}$, Valencia M. Tamaño y composición corporal en niños mexicanos: implicaciones en el uso del BOD, POD, DXA y dilución con deuterio en la evaluación de la masa grasa y masa libre de grasa. Rev Salud Publica y Nutrición. 2008;9(3):1-11.
8. McCarthy H, Cole T, Jebb S, Prenticem A. Pediatric Highlight: Body fat reference curves for children. Int J Obes. 2006;30:598-602.

9. Quintana E, Salas M, Cartín, M. Índice de masa corporal (IMC) y composición corporal con deuterio en niños costarricenses. Acta Pediátr Mex. 2014;35:179-189.

10. Innes $\mathrm{K}$, Vincent $\mathrm{H}$, Vincent $\mathrm{K}$. Oxidative stress and potential interventions to reduce oxidative stress in overweight and obesity. Diabetes Obesity Metabol. 2007;9:813-839.

11. Anagnostis $\mathrm{P}$, Athyros V, Tziomalos K, Karagiannis A, Mikhailidis D. The Pathogenetic Role of Cortisol in the Metabolic Syndrome: A Hypothesis. J Clin Endocrinol Metab. 2009;94(8):2692-2701.

12. Rebuffé $M$, Walsh $U$, McEwen B, Rodin J. Effect of chronic stress and exogenous glucocorticoid son regional fat distribution and metabolism. Physiol Behav.1992;52:583-590.

13. Duclos M, Marquez P, Barat P, Gatta B, Roger P. Increased cortisol bioavailability, abdominal obesity, and themetabolic syndrome in obese women. Obes Res Clin Pract. 2005;13:1157-1166.

14. Rask E, Olsson $T$, Soderberg $S$, Andrew R, Livingstone $D$, Johnson, O. Tissue-specific dysregulation of cortisol metabolism in human obesity. J Clin Endocrinol Metab. 2001;86:1418-1421.

15. Walker B, Soderberg S, Lindahl B, Olsson T. Independent effects of obesity and cortisol in predicting cardiovascular risk factors in men and women. J Intern Med. 2000;247:198-204.

16. Travison T, O'donnell A, Araujo A, Matsumoto A, McKinlay B. Cortisol levels and measures of body composition in middleaged and older men. Clin Endocrinol. 2007;67:71-77.

17. Stewart P, Krozowski Z.11ß-Hydroxysteroid dehydrogenase. Vitam Horm, 1999;57:249-324.

18. Espíndola D, Kater C. Adipose tissue expression of 11ß-hydroxysteroid dehydrogenase type 1 in Cushing's syndrome and in obesity. Arq Bras Endocrinol Metabol. 2007;51:1397-1403.

19. New M, Levine L, Biglieri E, Pareira J, Ulick S. Evidence for an unidentified steroid in a child with apparent mineralocorticoid hypertension. J Clin Endocrinol Metab. 1977;44:924-933.

20. Bishop M, Fody E, Schoeff L. Química Clínica Principios, Procedimientos y Correlaciones. 5arev. ed. México DF: McGraw-Hill; 2007.732p.

21. Holt H, Wild S, Postle A, Zhang J, Koster G, Umpleby M. Cortisol clearance and associations with insulin sensitivity, body fat and fatty liver in middle-aged men. Diabetologia. 2007;50:1024-1032.

22. Phillips D, Barker D, Fall C, Seckl J, Whorwood C, Wood P. Elevated plasma cortisol concentrations: a link between low birth weight and the insulin resistance syndrome?.J Clin Endocrinol Metab.1998;83:757-760. 
23. Reinehr T, Andler W. Cortisol and its relation to insulin resistance before and after weight loss in obese children. Horm Resist. 2004;62:107-112.

24. Misra M, Bredella M, Tsai P, Mendes N, Miller K, Klibanski A. Lowergrowth hormone and higher cortisol are associated with greater visceral adiposity, intramyocellular lipids, and insulin resistance in over weight girls. Am J Physiol Endocrinol Metab. 2008;295:E385-E392.

25. Delaunay F, Khan A, Cintra A, Davani B, Ling Z, Andersson A. Pancreaticcells are important targets for the diabetogenic effects of glucocorticoids. J Clin Invest. 1997;100:20942098.

26. Karauzene N, Mezouk H, Aribi M, Merzouk A, Berrouiguet A, Tessier C.Effects of theassociation of aging and obesityonlipids, lipoproteins and oxidative stress biomarkers: A comparison of older with young men. Nutr Metab Cardiovasc Dis. 2011;21:792-799.

27. Veldhuis J, Roemmich J, Richmond E, Rogol A, Lovejoy J, Sheffield M. Endocrine Control of Body Composition in Infancy, Childhood and Puberty. Endocr Rev. 2007;26(1):114-146.

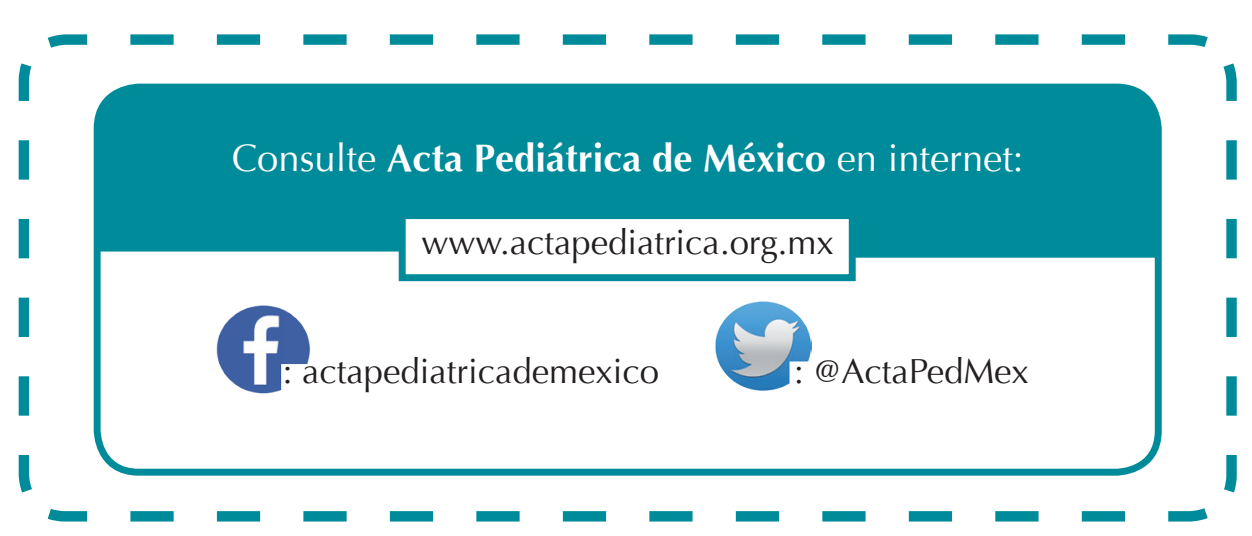

